\title{
Lorentz Transformation of Radiation 4-Potential
}

\author{
E. COMAY* \\ Charactell Ltd., PO Box 39019, Tel-Aviv, 61390, Israel
}

(Received August 6, 2017)

\begin{abstract}
The laws of Maxwellian electrodynamics are used in an analysis of the structure of the 4-potential of radiation fields. The paper examines multi-particle and single-particle effects of a radiating source. Causality of electromagnetic processes is an important element of the analysis. Covariance properties of the relevant variables are examined and the apparent non-covariance of the radiation 4-potential where $A_{0} \equiv 0$ in all frames is explained. It turns out that the origin of this feature stems from the multi-charge properties of radiation. It is also shown how in every Lorentz frame one can use covariant properties of radiation fields and reconstruct an appropriate 4-potential.
\end{abstract}

DOI: 10.12693/APhysPolA.133.1294

PACS/topics: covariance, 4-potential, electromagnetic radiation, electromagnetic fields, Lorentz invariants

\section{Introduction}

The main objective of this work is to apply well known laws of Maxwellian electrodynamics and derive a selfconsistent expression for the 4-potential of radiation. This expression satisfies two primary requirements of classical electrodynamics: the electromagnetic fields are the 4-curl of a 4-potential and the primary quantities undergo a self-consistent Lorentz transformation.

It is well known that electromagnetic radiation is derived from the vector potential $\boldsymbol{A}$ (see [1], p. 184 or [2], p. 391). It means that the 0 -component of a 4-potential $A_{0}$ vanishes for radiation fields and that this property holds for every Lorentz frame. Consequences of this aspect of the radiation 4-potential have already been pointed out in the literature. For example, Weinberg states in his textbook (see [3], p. 251): "The fact that $A^{0}$ vanishes in all Lorentz frames shows vividly that $A^{\mu}$ cannot be a four vector." An analogous statement can be found on p. 339 , where he says: "...the $A_{\mu}(x)$ transforms as a four-vector only up to a gauge transformation" (here $x$ denotes the four space-time coordinates). Indeed, the need for a gauge transformation is yet another proof of the claim that $A_{\mu}(x)$ does not transform as a 4 -vector simply because, by definition, a genuine 4 -vector transforms as a 4-vector without the need for a correction trick.

This case is an example of a general mathematical theorem stating that if a specific component of a tensor vanishes for all transformations then all components of this tensor vanish identically. This theorem can be easily proven for a 4 -vector where $A_{0}=0$ in all frames. Thus, assume that the theorem is incorrect and that there is a specific frame where the 4 -vector is

$$
A_{\mu}=(0, a, 0,0) \text {, }
$$

where $a \neq 0$. The metric is $\operatorname{diag}(1,-1,-1,-1)$ and units where the speed of light $c=1$ are used. Greek indices

*e-mail: elicomay@post.tau.ac.il run from 0 to 3 and the summation convention holds for a pair of upper and lower indices. Unless stated to the contrary, Latin indices run from 1 to 3 . Consider the following Lorentz transformation:

$$
L_{\nu}^{\mu}=\left(\begin{array}{llll}
\gamma & \beta & 0 & 0 \\
\beta & \gamma & 0 & 0 \\
0 & 0 & 1 & 0 \\
0 & 0 & 0 & 1
\end{array}\right),
$$

where $\gamma, \beta$ take real values, $\gamma>1, \beta \neq 0$ and

$$
\gamma^{2}-\beta^{2}=1
$$

(see [1], p. 9 or [2], p. 516). Applying the Lorentz transformation (2) to the 4 -vector (1) one obtains

$$
A_{\mu}^{\prime}=(a \beta, a \gamma, 0,0) \text {. }
$$

Hence, contrary to the primary assumption, $A_{0}^{\prime} \neq 0$ in the new frame. An analogous proof holds for cases where $A_{2} \neq 0$ or $A_{3} \neq 0$. This outcome completes the proof.

It is interesting to point out that covariance problems with the 4-potential are also found in the quantum theory where the canonical momentum associated with $A_{0}$ vanishes identically. Thus, it is stated that "it is at this point that we sacrifice manifest covariance..." (see [4], p. 71).

The main objective of this work is to show the reason for this outcome and to explain why, in spite of it, the Lorentz covariance is conserved in Maxwellian electrodynamics.

Multi-charge and single charge attributes of a radiating system are presented in the second and in the third sections, respectively. The fourth section shows how one can use covariant properties of electromagnetic fields and construct an acceptable 4-potential. The work is summarized in the last section.

\section{Multi-charge properties of radiation fields}

As stated in Introduction, the discussion relies on the well known laws of Maxwellian electrodynamics.The 
Lienard-Wiechert 4-potential of a charge $q$ is (see [1], p. 174 or [2], p. 656$)$ :

$$
A^{\mu}=q \frac{v^{\mu}}{R_{\alpha} v^{\alpha}} .
$$

Here $R^{\alpha}$ denotes the 4 -vector from the retarded spacetime position of the charge $x_{q}^{\alpha}$ to the field point $x^{\alpha}$ and $v^{\alpha}$ is the retarded 4-velocity of the charge. The electric and the magnetic fields of the charge $q$ are the 4-curl of the 4-potential (5) (see [1], p. 65 or [2], p. 550):

$$
F_{\mu \nu}=A_{\nu, \mu}-A_{\mu, \nu} .
$$

Here the fields tensor is (see [1], p. 65 or [2], p. 550):

$$
F^{\mu \nu}=\left(\begin{array}{cccc}
0 & -E_{x} & -E_{y} & -E_{z} \\
E_{x} & 0 & -B_{z} & B_{y} \\
E_{y} & B_{z} & 0 & -B_{x} \\
E_{z} & -B_{y} & B_{x} & 0
\end{array}\right) .
$$

Explicit expressions for the fields of (5) can respectively be written as a sum of two terms (see [1], p. 175 or [2], p. 657):

$$
\begin{aligned}
\boldsymbol{E} & =q \frac{1-v^{2}}{(R-\boldsymbol{R} \cdot \boldsymbol{v})^{3}}(\boldsymbol{R}-\boldsymbol{v} R) \\
& +\frac{q}{(R-\boldsymbol{R} \cdot \boldsymbol{v})^{3}} \boldsymbol{R} \times[(\boldsymbol{R}-\boldsymbol{v} R) \times \boldsymbol{a}]
\end{aligned}
$$

and

$$
\boldsymbol{B}=\boldsymbol{R} \times \boldsymbol{E} / R .
$$

Here $\boldsymbol{R}$ is the 3 -vector that is related to $R^{\mu}$ and $\boldsymbol{v}$ and $\boldsymbol{a}$ denote the charge's retarded velocity and acceleration, respectively. The first terms of (8) and of (9) are called velocity fields and the second ones are called acceleration fields.

The flux of electromagnetic energy is represented by the Poynting vector (see [1], p. 81 or [2], p. 237):

$$
\boldsymbol{S}=\boldsymbol{E} \times \boldsymbol{B} / 4 \pi \text {. }
$$

Relying on the inverse square law, one finds from the expression on the right hand side of (10) that radiation fields decrease like $1 / R$. It is clear that as the distance $R$ increases the first term of (8) decreases like $1 / R^{2}$ whereas the second term decreases like $1 / R$. Therefore, the first term of (8) and of (9) can be ignored at the radiation zone and the second terms of these expressions are the origin of radiation. The Poynting vector (10) has covariant properties because it is the $T^{0 i}$ components of the energy-momentum tensor $T^{\mu \nu}$ of the electromagnetic fields (see [1], p. 87 or [2], p. 605).

The three expressions (5), (8) and (9) abide by the principle of causality. Here one can see how properties of a charge $q$ at the retarded space-time point $x_{q}^{\mu}$ produce electromagnetic effects at the field space-time point $x^{\mu}$. Furthermore, the calculation of the retarded point of each charge (see [2], p. 655) shows that electromagnetic effects propagate at the speed of light.

It is interesting to point out another property of the 4-potential (5) and of the associated fields (8), (9). The expression for the fields consists of two terms which pertain to bound fields and radiation fields, respectively. On the other hand, one and the same 4-potential is used for the two kinds of fields. This feature explains why an analysis of radiation effects cannot be restricted to the 4-potentials but must also treat the fields (8), (9).

Let us examine the radiation emitted from the device depicted in Fig. 1A. Here the circle is embedded in the $(x, y)$ plane and the point $P$ is located on the $z$-axis at the radiation zone. The rotating charge $q$ accelerates towards the circle's center. Therefore, its acceleration fields do not vanish identically and the second terms of (8) and of (9) show that radiation fields exist at point $P$.

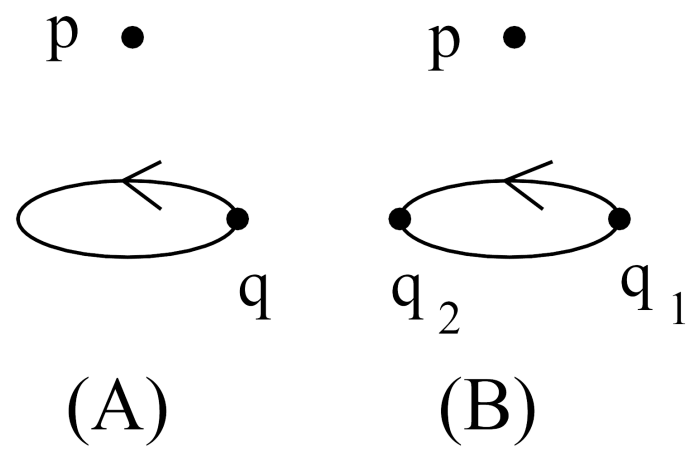

Fig. 1. Two radiating systems. (A) A charge $q$ moves uniformly along a circle. (B) Two equal charges move uniformly along a circle (See text.).

Now let us turn to the device depicted in Fig. 1B. Here are two equal charges $q_{1}=q_{2}=q$ which are located at two antipodal points of the circle. The charges rotate along the circle with the same velocity as that of the charge $q$ of Fig. 1A. Due to the antipodal position of the rotating charges, one finds that at every instant the values of the velocity and of the acceleration of the two charges satisfy $\boldsymbol{v}_{2}=-\boldsymbol{v}_{1}$ and $\boldsymbol{a}_{2}=-\boldsymbol{a}_{1}$. Similarly, for the point $P$ the retarded time of $q_{1}$ equals that of $q_{2}$.

Substituting these values in the second term of (8), one finds that in the case of Fig. 1B, the overall radiation field at point $P$ vanishes. One can also arrive at this conclusion from a general consideration. The system of Fig. 1B is invariant under a reflection of the $(x, y)$ coordinates. Hence, the $(x, y)$ components of the electric and of the magnetic fields at point $P$ vanish. Since the electromagnetic fields of radiation are perpendicular to the direction of the radiation, one finds that at point $P$ there is no radiation. A comparison between the two experiments depicted in Fig. 1 indicates that radiation is not a single particle property.

One can also push the effect to the extreme and examine the current that flows along a closed loop of a superconducting material or that of a direct current that is produced by a battery. Here charges accelerate but the system is time-independent. Therefore, no radiation is emitted by a system of this kind (see [1], p. 116).

A minor modification of Fig. 1B provides an alternative argument that proves the multi-charge property of radiation. Here the sign of the second charge is $q_{2}=-q_{1}$. 
As stated above, the acceleration of $q_{2}$ is $\boldsymbol{a}_{2}=-\boldsymbol{a}_{1}$. The opposite sign of the charge and of its acceleration means that at point $P$ the strength of each of the radiation fields $\boldsymbol{E}, \boldsymbol{B}$ doubles. Using the Poynting vector (10), one finds that the intensity of the radiation energy is four times greater that that of the radiation of Fig. 1A. Hence, also in this case one sees that radiation energy is not an arithmetic sum of the expression of the single-particle radiation terms.

Another argument that shows the multi-charge properties of radiation is the fact that there is no acceleration in a closed system which is made of a single charge.

The foregoing discussion shows that radiation is a multi-charge effect that is produced by all charges which compose the source.

\section{Single charge properties of the 4-potentials}

Let us return to the Lienard-Wiechert 4-potential (5). This expression determines the contribution of a single charge $q$. Due to the linearity of the Maxwell equations, one may sum the contribution of all charges and obtain an expression for the total 4-potential of the system

$$
A_{\text {Total }}^{\mu}=\sum_{i} q_{i} \frac{v_{i}^{\mu}}{R_{i \alpha} v_{i}^{\alpha}},
$$

where the index $i$ runs on all charges of the radiating system.

The purpose of the analysis carried out in this work is to find out properties of electromagnetic radiation. As shown in Sect. 2, these properties pertain to the fields (8), (9) which are derived from the 4-potential (5). An observation of (11) shows clearly that the fields which are obtained from the 4-curl of this 4-vector depend on the retarded variables of each charge. (A calculation of these fields can be found on p. 10 of [5].) As a matter of fact, details of this calculation are not required here, because it is enough to realize that for different charges the retarded 4 -velocity and its associated retarded 4-acceleration generally take quite different values. Furthermore, the retarded time of any two charges is generally not the same, because each charge moves at the source's region along its own world line. For this reason, one must separately differentiate each term that stands on the right hand side of (11). This conclusion shows a single particle property of a radiating system of charges.

Let us examine the Lorentz transformations of the Lienard-Wiechert 4-potentials (11). Evidently, each term $i$ of the right hand side of (11) takes the form of the 4 -vector $v_{i}^{\mu}$ multiplied by the charge $q_{i}$ which is a Lorentz scalar and divided by $R_{i \alpha} v_{i}^{\alpha}$ which is also a Lorentz scalar. Hence, by general laws of tensor algebra, the left hand side of (11) is a 4-vector. However, for a given field point $x^{\mu}$, each charge has its own retarded time, and at this instant, its 4 -velocity is multiplied by a positive or a negative charge and divided by a specific scalar. Furthermore, it is pointed out in the second section that in order to extract the radiation component of the system, one must examine the fields (8) and (9) which are the 4-curl of the 4-potential. It follows that in order to preserve the possibility of taking the 4-curl of the 4-potentials (11), the Lorentz transformation should be applied to the individual terms that stand on the right hand side of (11). For this reason, a single term that stands on the left hand side of (11) cannot be used as a 4-potential because a derivation of the fields is obtained from the 4-curl of the individual terms that stand on the right hand side of (11). This operation depends on the specific values of the retarded velocity and the retarded acceleration of each charge.

The right hand side of (11) also shows how the principle of causality works in the case of radiation fields. Here physical properties of each charge at its specific instant in the past contribute to an effect which takes place in the present at a laboratory space time point $x^{\mu}$. Evidently, the retarded variables of each charge of the system make their own contribution.

This section explains why a single 4-vector cannot describe properly the 4-potential of radiation fields.

\section{Covariance of an effective 4-potential for radiation fields}

It is important to emphasize the significant role of the electromagnetic fields and of their 4-potential in a description of the electromagnetic part of a given system. For this purpose let us examine the electromagnetic terms of the Lagrangian density (see [1], p. 75 or [2], p. 596):

$$
\mathcal{L}_{E M}=-\frac{1}{16 \pi} F^{\mu \nu} F_{\mu \nu}-j^{\mu} A_{\mu},
$$

where $j^{\mu}$ denotes the 4 -current of the electric charge. This expression proves that the first term shows how the fields tensor $F^{\mu \nu}$ describes the existence of an electromagnetic entity and the second term shows how this entity is measured by means of the coupling of its 4potential $A_{\mu}$ with the 4 -current $j^{\mu}$ of an electric charge. A corresponding expression is used for quantum fields (see [3], p. 349 or [6], p. 78), and in the case of a Dirac particle the charge's 4-current is $j^{\mu}=e \bar{\psi} \gamma^{\mu} \psi$ (see [3], p. 355 or [6], p. 50).

Conclusion: Both the fields and their 4-potential are required for a description of the behavior of an electromagnetic system.

Radiation energy (a photon in a quantum parlance) is an objective electromagnetic entity. However, the discussion of the previous section explains why a single term cannot describe covariantly the 4-potential of radiation fields. Due to the non-covariance of the radiation 4potential which is proved above, it is required to construct an effective 4-potential that will be used in theories that are derived from the variational principle in general, and in quantum theories in particular. Evidently, for every given Lorentz frame, this 4-potential must be consistent with the fields. 
Let us examine the Lorentz invariants of the electromagnetic fields (see [1], p. 68)

$$
\operatorname{Inv}_{1}=B^{2}-E^{2}
$$

and

$$
\mathrm{Inv}_{2}=\boldsymbol{E} \cdot \boldsymbol{B} .
$$

An examination of the radiation term of the electromagnetic fields (8) and (9) indicates that these invariants vanish for radiation fields emitted from a specific source. This result is also obtained from (66.3) of [1], p. 185. Furthermore, the Poynting vector (10), which shows the direction of the fields' momentum density, proves that radiation fields are perpendicular to the direction of propagation. These properties hold for every inertial frame and for this reason they represent a covariant property of radiation fields. They are written here for a further reference

$$
\boldsymbol{E} \perp \boldsymbol{B}, \quad \boldsymbol{E} \perp \boldsymbol{p}, \quad \boldsymbol{B} \perp \boldsymbol{p},
$$

where $\boldsymbol{p}$ denotes the Poynting vector.

The covariance of (15) helps us understand the relativistic meaning of the standard 4-potential of radiation fields. Here the 4-potential is not a 4 -vector which is defined in terms of the retarded quantities of the source but it is reconstructed in every Lorentz frame by means of covariant fields that are measured at the laboratory. It is shown below how relations (15) are used in a construction of a specific expression for the required 4-potential. In particular, this 4 -potential satisfies $A_{0} \equiv 0$ in every Lorentz frame.

Let us assume that at the laboratory the radiation propagates parallel to the $z$-axis and examines its Fourier component $\exp (i(k z-\omega t))$, where its wavelength is $\lambda=$ $2 \pi / k$. Taking advantage of the following property of the radiation zone:

$$
\lambda \ll R,
$$

where $R$ denotes the distance from the laboratory point $x^{\mu}$ to the radiation source (see [2], p. 392), one may regard the radiation at the laboratory as a plane wave.

This discussion boils down to the following expression for the Fourier component of the 4-potential of the radiation which is polarized in the $x$-direction:

$$
\boldsymbol{A}(z, t)=\left(A_{x} \exp i(k z-\omega t), 0,0\right) .
$$

Here only the $x$-component of the vector potential $\boldsymbol{A}(z, t)$ does not vanish and it is parallel to the electric field $\boldsymbol{E}$. An analogous expression holds for a wave which is polarized in the $y$-direction. Unlike the Lienard-Wiechert 4potential (5) which depends explicitly on retarded quantities, the radiation potential (17) depends on quantities at the field point $x^{\mu}$ and has no explicit dependence on retarded quantities of individual charges at the source. The explicit dependence of the potential (17) on local space-time coordinate shows that it is suitable for the expression that describes the charge-fields interaction (12).

The results of this section can be summarized in the following words. The electromagnetic fields (7) transform covariantly. Furthermore, the perpendicularity relations (15) of radiation fields are covariant properties. There- fore, covariant attributes of the 4-potential are seen if it is not regarded as an independent 4-vector but as a quantity that is reconstructed in every frame on the basis of covariant fields.

\section{Conclusions}

The following points summarize the results of this work.

- Interference of electromagnetic fields proves that the radiation emitted from a system is a multicharge effect where every charge makes its own contribution.

- At every field point, each charge has its own 4potential which is determined by the LienardWiechert expression (5).

- Each charge makes its own contribution to the interfering fields. These fields depend on the retarded velocity and the retarded acceleration of the charges at the source.

- Each charge has its own retarded velocity and acceleration. Therefore, the fields are obtained from a separate calculation of the 4-curl of the retarded 4-potential of each charge. Only after this task is accomplished these fields are summed up and specific interference effects are obtained.

- The separate treatment of the 4-potential of each charge shows how the principle of causality works for electromagnetic radiation.

- A single covariant term that is the sum of the 4potentials of the charges cannot be used for deriving the fields because of the different values of the retarded velocity and acceleration of the charges.

- A Lorentz transformation of the 4-potential should be separately performed for each term that stands on the right hand side of (11). This requirement stems from the dependence of the fields on the retarded velocity and on the associated acceleration of each charge.

- An effective expression for the 4-potential can be constructed in a covariant form. Here the covariance of the fields shows that this 4-potential can be reconstructed in every Lorentz frame. The 0component of this 4-potential vanishes identically.

This work shows the interrelations between the global effect of radiation and the contribution which is made by individual charges. The 4-potential and its associated fields are obtained from a separate calculation of the dynamics of each charge and only after this task is finished the fields are summed up and yield the appropriate interference effects. For every frame, an acceptable 4-potential (which is not a 4-vector) can be constructed from covariant properties of radiation fields. 


\section{References}

[1] L.D. Landau, E.M. Lifshitz, The Classical Theory of Fields, Elsevier, Amsterdam 2005.

[2] J.D. Jackson, Classical Electrodynamics, Wiley, New York 1975

[3] S. Weinberg, The Quantum Theory of Fields, Vol. I, Cambridge University Press, Cambridge 1995.
[4] J.D. Bjorken, S.D. Drell, Relativistic Quantum Fields, McGraw-Hill, New York 1965.

[5] C. Teitelboim, D. Villarroel, Ch.G. van Weert, Riv. Nuovo Cimento 3, 1 (1980).

[6] M.E. Peskin, D.V. Schroeder, An Introduction to Quantum Field Theory, Addison-Wesley, Reading Mass. 1995. 